\section{A fluctuation theorem in a random environment}

\author{
F.Bonetto, G.Gallavotti, G.Gentile \\ Mathematics, Georgia Tech., Atlanta \\ I.N.F.N. Roma 1, Fisica Roma1 \\ Matematica, Università di Roma 3
}

Abstract: A simple class of chaotic systems in a random environment is considered and the fluctuation theorem is extended under the assumption of reversibility.

\section{Random chaos}

A chaotic system in random environment is defined by

(1) an environment which can be in states $\boldsymbol{\omega}$ belonging to a space $\Omega$ and varying in time; the time evolution being given by a map $\tau$ on $\Omega$;

(2) a $\tau$-invariant probability distribution $P$ on $\Omega$ describing the statistical properties of the environment evolution;

(3) a family of maps $x \rightarrow f_{\boldsymbol{\omega}}(x)$ of a manifold $\mathcal{F}$ (phase space) into itself.

The space of the random events $\Omega$ will be supposed a space of sequences of finitely many symbols $\boldsymbol{\omega}=$ $\left\{\omega_{j}\right\}_{j=-\infty}^{\infty}$, for instance a sequence of spins $\omega_{j}= \pm 1$, with the usual metric $d\left(\boldsymbol{\omega}, \boldsymbol{\omega}^{\prime}\right)=\sum_{j}\left|\omega_{j}-\omega_{j}^{\prime}\right| 2^{-|j|}$, and with $\tau$ the shift to the left: $(\tau \boldsymbol{\omega})_{j}=\omega_{j+1}$. A "reflection" operator will be defined on $\Omega$ as $\boldsymbol{\omega} \longleftrightarrow \boldsymbol{\omega}^{T}$, with $\left(\boldsymbol{\omega}^{T}\right)_{j}=\omega_{-j}$. The probability distribution $P$ will be a $\tau$-invariant and reflection-invariant mixing process on $\Omega$, for instance a Bernoulli shift or a Markov process or a general Gibbs distribution on $\Omega$. Reflection-invariant means that $P(E) \equiv P\left(E^{T}\right)$ for every Borel set $E$, where $E^{T}$ is the image of $E$ under the reflection $\boldsymbol{\omega} \longleftrightarrow \boldsymbol{\omega}^{T}$. The manifold $\mathcal{F}$ will be a torus $\mathbb{T}^{m}$ and $f_{\boldsymbol{\omega}}$ will be supposed to be close to a map $f$ which is a "linear hyperbolic torsion" of $\mathbb{T}^{m}$, independent of the environment, i.e. close to a map which is defined by the action of a matrix with integer entries and determinant 1 on the torus.

For instance the point $x \in \mathcal{F}$ can be visualized as a pair of clock arms $x=\varphi=\left(\varphi_{1}, \varphi_{2}\right)$ frantically moving as

$$
\varphi \rightarrow S_{0}(\varphi)=\left(\varphi_{1}+\varphi_{2}, \varphi_{1}\right)
$$

The map is a "linear hyperbolic torsion" of $\mathbb{T}^{2}$ and is a paradigmatic example of Anosov maps.

The restriction on the choice of $f$, however, is not dictated by a simplicity requirement alone. The present technique is very special for the cases that we shall consider. However it should be possible to extend them to a more general situation using the ideas in [1].

The actual $f_{\boldsymbol{\omega}}$ will be small perturbations of $f$. For simplicity, for a small $\varepsilon$, we will take it of the form

$$
x \rightarrow f_{\boldsymbol{\omega}}(x)=f(x)+\varepsilon \omega_{0} \psi(x),
$$

where $\omega_{0} \in\{-1,1\}$ and $\psi$ is a trigonometric polynomial periodic on $\mathbb{T}^{m}$. Therefore we are led to consider the dynamical system $\left(\Omega \times \mathcal{F}, \mathcal{S}_{\varepsilon}\right)$, with

$$
\mathcal{S}_{\varepsilon}(\boldsymbol{\omega}, x)=\left(\tau \boldsymbol{\omega}, f_{\boldsymbol{\omega}}(x)\right) .
$$

In other words at every instant $t$ the next coin is flipped (or the next spin state is observed) and the system point $x_{t}$ is moved by $f\left(x_{t}\right)+\varepsilon \omega_{t} \psi\left(x_{t}\right)$. We shall denote $\mathbf{x}=$ $(\boldsymbol{\omega}, x)$ the points in $\Omega \times \mathcal{F}$.

According to standard terminology if $\mu_{0}$ denotes the normalized volume measure on $\mathcal{F}$ ("Liouville measure") the system will be said to possess a well defined statistics if, for almost all $(\boldsymbol{\omega}, x) \in \Omega \times \mathcal{F}$ in the $P \times \mu_{0}$-distribution sense, the limit

$$
\lim _{T \rightarrow \infty} \frac{1}{T} \sum_{j=0}^{T-1} F\left(f_{\boldsymbol{\omega}}^{* j}(x)\right)=\int_{\mathcal{F}} \mu(\mathrm{d} y) F(y)
$$

exists for all continuous "observables" $F$ on $\mathcal{F}$ (hence for many others), and is independent of $\mathbf{x}=(\boldsymbol{\omega}, x)$, thus defining a probability distribution $\mu$ which will be called the statistics of the motion or the SRB distribution; here $f_{\omega}^{* j}(x), j \in \mathbb{Z}$ is defined as the composition of $j$ maps

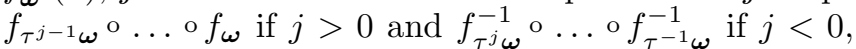
and $f_{\boldsymbol{\omega}}^{0}=1$. More ambitiously one could look for an $\mathcal{S}_{\varepsilon}$-invariant distribution of the form

$$
\mu_{\mathrm{srb}}(\mathrm{d} \boldsymbol{\omega} \mathrm{d} x)=P(\mathrm{~d} \boldsymbol{\omega}) \mu_{\boldsymbol{\omega}}(\mathrm{d} x),
$$

with $\lim _{T \rightarrow \infty} \frac{1}{T} \sum_{j=0}^{T-1} F\left(\mathcal{S}_{\varepsilon}^{j}(\boldsymbol{\omega}, x)\right)$ existing for all continuous observables $F$ on $\Omega \times \mathcal{F}$, apart from a 0 probability set with respect to $P \times \mu_{0}$, and having the form $\int_{\boldsymbol{\Omega} \times \mathcal{F}} F\left(\boldsymbol{\omega}^{\prime}, y\right) \mu_{\mathrm{srb}}\left(\mathrm{d} \boldsymbol{\omega}^{\prime} \mathrm{d} y\right) \stackrel{\text { def }}{=}\langle F\rangle_{\mathrm{srb}}$; when existing, $\mu_{\text {srb }}$ is also called the SRB distribution and it is related to (1.4) by $\mu(\mathrm{d} x)=\int_{\Omega} \mu_{\boldsymbol{\omega}}(\mathrm{d} x) P(\mathrm{~d} \boldsymbol{\omega})$.

Systems more general than the above have been considered in [2] where

$$
\sigma_{\boldsymbol{\omega}}(x)=-\log \left|\operatorname{det} \frac{\partial f_{\boldsymbol{\omega}}(x)}{\partial x}\right|
$$

is introduced and called entropy production rate: furthermore it is proved that $\sigma_{+} \stackrel{\text { def }}{=}\langle\sigma\rangle_{\text {srb }} \geq 0$, see (1.5). Here we shall mainly consider the case $\sigma_{+}>0$, which is the generic case.

Particular attention will be given to reversible systems: namely systems for which there is a smooth map $\mathcal{I}$ : $\mathcal{I}(\boldsymbol{\omega}, x)=\left(\boldsymbol{\omega}^{\prime}(\boldsymbol{\omega}), x^{\prime}(\boldsymbol{\omega}, x)\right)$, with $\boldsymbol{\omega}^{\prime}(\boldsymbol{\omega}) \stackrel{\text { def }}{=} \boldsymbol{\omega}^{T}$, such that $\mathcal{I}^{2}= \pm 1$ and

$$
\mathcal{I} \circ \mathcal{S}_{\varepsilon}^{k}=\mathcal{S}_{\varepsilon}^{-k} \circ \mathcal{I}
$$

for some integer $k$. 
Examples of such systems are rather simple to give in the non-random case: however in the random cases (like the ones considered here) the very existence of $\mathcal{I}$ is not obvious.

In this paper we follow the general ideas of the "cluster expansion" techniques which were introduced in [3-7] but developed following the tree expansion method of [1], see also [8].

\section{Fluctuation theorem}

In the non-random case, i.e. if $f_{\boldsymbol{\omega}}=f$ is independent of $\boldsymbol{\omega}$, and for $f$ a general Anosov map it has been shown that a time reversal symmetry can be translated into certain relations between the probabilities of the "large fluctuations" of the time averages of the dimensionless observable $\sigma_{\boldsymbol{\omega}}(x) / \sigma_{+}$. Namely consider the observable

$$
p(x)=p(\mathbf{x}) \stackrel{\text { def }}{=} \frac{1}{T} \sum_{j=0}^{T-1} \frac{\sigma_{\tau^{j} \boldsymbol{\omega}}\left(f_{\boldsymbol{\omega}}^{* j} x\right)}{\sigma_{+}},
$$

and call $\pi_{T}(p \in \Delta)$ the probability that it takes value in an interval $\Delta$ evaluated in the stationary SRB distribution (which for Anosov maps exists). Then $\pi_{T}(p \in$ $\Delta)=e^{T \zeta(p)+O(1)}$ where $\zeta(p)$ is an analytic function of $p$ defined in the interval $\left(-p^{*}, p^{*}\right)$, for some $p^{*}>1$. The time reversal symmetry implies

$$
\zeta(-p)=\zeta(p)-p \sigma_{+}, \quad|p|<p^{*},
$$

which is called the fluctuation theorem, and was proven in $[9-11]$.

The above statements concern non-random maps (i.e. $f_{\boldsymbol{\omega}}=f$ is $\boldsymbol{\omega}$ independent). This note is dedicated to show that (2.2) also holds for the random noise cases introduced in (1.3) with $f_{\boldsymbol{\omega}}$ given by (1.2) and $f=S_{0}$, as given for instance by (1.1), when a time reversal symmetry, (1.7), $\mathcal{I}(\boldsymbol{\omega}, x)=\left(\boldsymbol{\omega}^{\prime}(\boldsymbol{\omega}), x^{\prime}(\boldsymbol{\omega}, x)\right.$ holds with $\boldsymbol{\omega}^{\prime}(\boldsymbol{\omega}) \stackrel{\text { def }}{=} \boldsymbol{\omega}^{T}=\left\{\boldsymbol{\omega}_{-j}\right\}_{j=-\infty}^{\infty}$ and $P$ is invariant for the $\operatorname{map} \boldsymbol{\omega} \longleftrightarrow \boldsymbol{\omega}^{T}$.

A proof can be achieved by trying to fall back on the already existing proofs of extensions of the fluctuation theorem to stochastic processes; the first one in [12] has been followed and widely extended in $[13,14]$.

In the first examples mentioned at the beginning of Sec.1, in which the $\boldsymbol{\omega}$ 's are a sequence of \pm 1 with independent distribution or with finite range coupling, it is clear that the process $\mu(\mathrm{d} \boldsymbol{\omega} \mathrm{d} x)$ is a Markov process and the analysis in Sec.2 of [13] can in principle be applied. In the present case the phase space is a continuum but the results in the quoted paper nevertheless apply, at least if one is willing to consider formally ratios of delta functions and interpret them as suitable Jacobian determinants.

To discuss the example of a general Gibbs distribution, in which the distribution of the $\boldsymbol{\omega}$ is far from Markovian, along the lines of [13] is harder. But one can consider the more general approach in [14]. However in this case one has to prove that the SRB distribution is a "space-time Gibbs state", which is the basic object studied in [14].

In all cases an explicit determination of the stationary state seems necessary in order to check the assumptions and to define and compute the quantity $e(\lambda)$ of the quoted references. In other words the existing literature provides strong arguments (particularly in the Markovian cases) for the validity of a relation like (2.1): but some work to check it remains to be done, on a case by case basis, and a substantial further work is necessary if one wishes to compute the function $\zeta(p)$ or at least some of its properties.

We restrict attention to the (very special) cases

(1) $\mathcal{S}_{\varepsilon}$ defined by $(1.2),(1.3),(1.1)$ and

(2) $\psi(x)$ trigonometric polynomial.

We shall show that the technique of [15] can be applied to obtain, for $\varepsilon$ small enough, the results:

(i) the $S R B$ distribution $\mu$ exists and is unique;

(ii) it is a space-time Gibbs distribution;

(iii) $\sigma_{+}>0$ at least for $\varepsilon \neq 0$ small enough;

(iv) under the extra assumption of existence of a time reversal symmetry for $\mathcal{S}$, the large deviation rate $\zeta(p)$ for $p(x)$, see (2.1), satisfies the symmetry relation (2.2), i.e. a fluctuation theorem holds.

Remarks: (i) The extension of the results to perturbations of torsions of tori of arbitrary dimension is not treated because it is identical to the one we present here but the notational burden hides the simplicity of the ideas.

(2) The apparent restriction to perturbations of Anosov maps generated by a linear torsion of a torus is due to the use made of the flatness and parallelism properties of the stable (respectively unstable) planes for such torsions. However by applying the methods in [1] the results can be extended to the general case in which perturbations of generic analytic Anosov maps of tori of arbitrary dimension are considered, see comments in Sec.8.

\section{Decoupling and shadowing}

The key for fulfilling the program set in Sec.2 is to show that there is a map $\mathbf{x}^{\prime} \equiv\left(\boldsymbol{\omega}^{\prime}, x^{\prime}\right) \longleftrightarrow \mathbf{x} \equiv(\boldsymbol{\omega}, x)$, that we call $H_{\varepsilon}$, of the form

$$
\left(\boldsymbol{\omega}^{\prime}, x^{\prime}\right)=H_{\varepsilon}(\boldsymbol{\omega}, x) \stackrel{\text { def }}{=}\left(\boldsymbol{\omega}, x+h_{\boldsymbol{\omega}}(x)\right),
$$

with $h_{\boldsymbol{\omega}}(x)$ analytic in $\varepsilon$ (but not in $x$, in general) such that

$$
\mathcal{S}_{\varepsilon} \circ H_{\varepsilon}=H_{\varepsilon} \circ \mathcal{S}_{0}
$$

where $\mathcal{S}_{0}(\boldsymbol{\omega}, x)=\left(\tau \boldsymbol{\omega}, S_{0} x\right)$. This means that there is a change of variables turning the perturbed map into the unperturbed one, i.e. the perturbed map $\mathcal{S}_{\varepsilon}$ can be "conjugated" to the unperturbed one $\mathcal{S}_{0}$. The relation (3.2) becomes, see (1.2),

$$
S_{0} h_{\boldsymbol{\omega}}(x)-h_{\tau \boldsymbol{\omega}}\left(S_{0} x\right)=-\varepsilon \psi_{\boldsymbol{\omega}}\left(x+h_{\boldsymbol{\omega}}(x)\right),
$$


where $S_{0}$ is a $2 \times 2$ matrix in our case.

We look for a solution which is analytic in $\varepsilon: h_{\boldsymbol{\omega}}(x)=$ $\varepsilon h_{\boldsymbol{\omega}}^{(1)}(x)+\varepsilon^{2} h_{\boldsymbol{\omega}}^{(2)}(x)+\cdots$, with $h_{\boldsymbol{\omega}}^{(k)}(x) \varepsilon$-independent functions. For instance the equation for the first order is

$$
S_{0} h_{\boldsymbol{\omega}}^{(1)}(x)-h_{\tau \boldsymbol{\omega}}^{(1)}\left(S_{0} x\right)=-\psi_{\boldsymbol{\omega}}(x) .
$$

Call $v_{ \pm}$the two normalized eigenvectors of $S$ relative to the eigenvalues $\lambda_{ \pm}$and let $\lambda$ the inverse of the largest one $\left(\lambda=\frac{1}{2}(\sqrt{5}-1)\right)$, so that $\lambda_{+}=\lambda^{-1}, \lambda_{-}=-\lambda: \lambda<1$.

The functions $\psi_{\boldsymbol{\omega}}, h_{\boldsymbol{\omega}}$ can be split into two components along the vectors $v_{ \pm}$:

$$
\begin{gathered}
\psi_{\boldsymbol{\omega}}(x)=\psi_{\boldsymbol{\omega},+}(x) v_{+}+\psi_{\boldsymbol{\omega},-}(x) v_{-}, \\
h_{\boldsymbol{\omega}}(x)=h_{\boldsymbol{\omega},+}(x) v_{+}+h_{\boldsymbol{\omega},-}(x) v_{-},
\end{gathered}
$$

and the equation (3.5) for $h_{\boldsymbol{\omega}, \pm}^{(1)}$ gives

$$
\begin{aligned}
& \lambda_{+} h_{\boldsymbol{\omega},+}^{(1)}(x)-h_{\tau \boldsymbol{\omega},+}^{(1)}\left(S_{0} x\right)=\psi_{\boldsymbol{\omega},+}(x), \\
& \lambda_{-} h_{\boldsymbol{\omega},-}^{(1)}(x)-h_{\tau \boldsymbol{\omega},-}^{(1)}\left(S_{0} x\right)=\psi_{\boldsymbol{\omega},-}(x),
\end{aligned}
$$

which can be solved by simply setting

$$
h_{\boldsymbol{\omega}, \alpha}^{(1)}(x)=-\sum_{p \in \mathbb{Z}_{\alpha}} \alpha \lambda_{\alpha}^{-|p+1| \alpha} \psi_{\tau^{p} \boldsymbol{\omega}, \alpha}\left(S_{0}^{p} x\right),
$$

where $\alpha= \pm, \mathbb{Z}_{+}=[0, \infty) \cap \mathbb{Z}, \mathbb{Z}_{-}=(-\infty, 0) \cap \mathbb{Z}$ and the inequality $\lambda<1$ ensures convergence.

Hence the equations for $h_{ \pm}^{(k)}$ become

$$
\begin{aligned}
& h_{\boldsymbol{\omega}, \alpha}^{(k)}(x)=-\sum_{s=0}^{\infty} \frac{1}{s !} \sum_{\substack{k_{1}+\cdots+k_{s}=k-1, k_{i} \geq 0 \\
\alpha_{1}, \ldots, \alpha_{s}= \pm}} . \\
& \cdot \sum_{p \in \mathbb{Z}_{\alpha}} \alpha \lambda_{\alpha}^{-|p+1| \alpha}\left(\prod_{j=1}^{s}\left(v_{\alpha_{j}} \cdot \partial_{x}\right)\right) \psi_{\tau^{p} \boldsymbol{\omega}, \alpha}\left(S_{0}^{p} x\right) . \\
& \cdot\left(\prod_{j=1}^{s} h_{\tau^{p} \boldsymbol{\omega}, \alpha_{j}}^{\left(k_{j}\right)}\left(S_{0}^{p} x\right)\right) .
\end{aligned}
$$

This can be written via a graphical representation as in Fig.1, where the "graph element" on the 1.h.s. represents $h_{\boldsymbol{\omega}, \alpha}^{(k)}(x)$. Representing again, in the same way, the graph elements that appear on the r.h.s. one obtains an expression for $h_{\boldsymbol{\omega}, \alpha}^{(k)}(x)$ in terms of trees, oriented "toward the root".

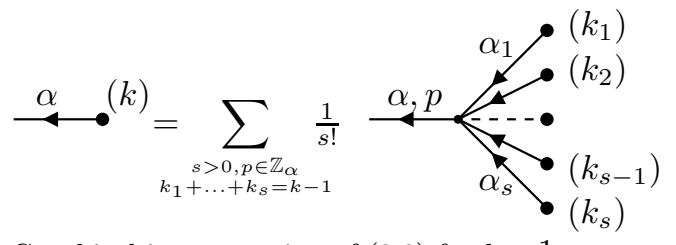

Fig.1 Graphical interpretation of (3.8) for $k>1$.

A tree $\theta$ with $k$ nodes will carry on the branches $\ell$ a pair of labels $\alpha_{\ell}, p_{\ell}$, with $p_{\ell} \in \mathbb{Z}$ and $\alpha_{\ell} \in\{-,+\}$, and on the nodes $v$ a pair of labels (not shown in Fig.1) $\alpha_{v}, p_{v}$, with $\alpha_{v}=\alpha_{\ell_{v}}$ and $p_{v} \in \mathbb{Z}_{\alpha_{v}}$ such that

$$
p(v) \equiv p_{\ell_{v}}=\sum_{w \succeq v} p_{v}
$$

where the sum is over the nodes following $v$ (i.e. over the nodes along the path connecting $v$ to the root), $\ell_{v}$ denotes the branch exiting from the node $v$, and to each tree we shall assign, given $\boldsymbol{\omega}$, a value given by

$$
\begin{aligned}
\operatorname{Val}(\theta)= & \prod_{v \in V(\theta)} \frac{-\alpha_{v}}{s_{v} !} \lambda_{\alpha_{v}}^{-\left|p_{v}+1\right| \alpha_{v}} . \\
& \cdot\left(\prod_{j=1}^{s_{v}} \partial_{\alpha_{v_{j}}}\right) \psi_{\tau^{p(v)} \boldsymbol{\omega}, \alpha_{v}}\left(S_{0}^{p(v)} x\right),
\end{aligned}
$$

where $\partial_{\alpha} \stackrel{\text { def }}{=} v_{\alpha} \cdot \partial_{x}, V(\theta)$ is the set of nodes in $\theta$, the nodes $v_{1}, \ldots, v_{s_{v}}$ are the $s_{v}$ nodes preceding $v$ (if $v$ is a top node then the derivatives are simply missing). If $\Theta_{k, \alpha}$ denotes the set of all trees with $k$ nodes and with label $\alpha$ associated with the root line, then one has

$$
h_{\boldsymbol{\omega}, \alpha}(x)=\sum_{k=1}^{\infty} \varepsilon^{k} \sum_{\theta \in \Theta_{k, \alpha}} \operatorname{Val}(\theta)
$$

and the "only" problem left is to estimate the radius of convergence of the above formal power series. For this purpose it is convenient to study the Fourier transform of the function $h_{\boldsymbol{\omega}, \alpha}(x)$. This is easily done graphically because it is enough to attach a label $\boldsymbol{\nu}_{v} \in \mathbb{Z}^{2}$ to each node and define the momentum that "flows" on the tree branch $\ell_{v}$ as $\boldsymbol{\nu}_{\ell_{v}} \stackrel{\text { def }}{=} \sum_{w \preceq v} \boldsymbol{\nu}_{w}$.

Then (3.11) becomes

$$
h_{\boldsymbol{\omega}, \alpha}(x)=\sum_{k=1}^{\infty} \varepsilon^{k} \sum_{\boldsymbol{\nu} \in \mathbb{Z}^{2}} e^{i \boldsymbol{\nu} \cdot x} h_{\boldsymbol{\omega}, \alpha, \boldsymbol{\nu}}^{(k)}
$$

with

$$
\begin{aligned}
& h_{\boldsymbol{\omega}, \alpha, \boldsymbol{\nu}}^{(k)}=\sum_{\theta \in \Theta_{k, \boldsymbol{\nu}, \alpha}} . \\
& \cdot\left(\prod_{v \in V(\theta)} \frac{-\alpha_{v}}{s_{v} !} \lambda_{\alpha_{v}}^{-\left|p_{v}+1\right| \alpha_{v}} \psi_{\tau^{p(v)} \boldsymbol{\omega}, \alpha_{v}, S_{0}^{-p(v)} \boldsymbol{\nu}_{v}}\right) . \\
& \cdot \prod_{\substack{v \in V(\theta) \\
v^{\prime} \neq v_{0}}}\left(i S_{0}^{-p\left(v^{\prime}\right)} \boldsymbol{\nu}_{v^{\prime}} \cdot v_{\alpha_{v}}\right)
\end{aligned}
$$

where $\Theta_{k, \boldsymbol{\nu}, \alpha}$ denotes the set of all trees with $k$ nodes and with labels $\boldsymbol{\nu}$ and $\alpha$ associated with the root line, and $v_{0}$ is the node immediatley preceding the root.

Since we are assuming that $\psi_{\boldsymbol{\omega}}$ is a trigonometric polynomial of degree $N$ and with uniformly bounded coefficients as $\boldsymbol{\omega}$ varies we can define $F=\max _{\boldsymbol{\nu}}\left|\psi_{\boldsymbol{\omega}, \boldsymbol{\nu}}\right|$. Then, to study the regularity of the conjugation, we shall estimate $\sum_{\boldsymbol{\nu}}|\boldsymbol{\nu}|^{\beta}\left|h_{\alpha, \nu}^{(k)}\right|$ for $\beta \geq 0$. The only problem is given by the presence of the factor $|\boldsymbol{\nu}|^{\beta}$. In fact consider first the case $\beta=0$ : there are only $(2 N+1)^{2}<(3 N)^{2}$ possible choices for each $\boldsymbol{\nu}_{v}$, given $p(v)$, such that $\left|S_{0}^{-p(v)} \boldsymbol{\nu}_{v}\right| \leq N$. Hence fixed $\theta,\left\{\alpha_{v}\right\}_{v \in V(\theta)}$ and $\left\{p_{v}\right\}_{v \in V(\theta)}$ the remaining sum of products in (3.13) is bounded by (if $\lambda \equiv \lambda_{+}^{-1} \equiv$ 
$\left.-\lambda_{-}\right)$

$$
(3 N)^{2 k} N^{k} F^{k} \prod_{v \in V(\theta)} \frac{\lambda^{\left|p_{v}+1\right|}}{s_{v} !}
$$

having bounded by $N^{k}$ the last product in (3.13). The sum over the $\left\{p_{v}\right\}_{v \in V(\theta)}$ is a multiple geometric series bounded by $(2 /(1-\lambda))^{k}$.

The combinatorial factors arising from the Taylor expansion leading to (3.8) (see the $s !^{-1}$ factors in (3.8) or the $s_{v} !^{-1}$ factors in (3.14)) compensate the large number of trees so that the factor $\prod_{v}\left(1 / s_{v}\right.$ !) becomes, after summing over all the trees, simply bounded by $2^{3 k},\left(2^{2 k}\right.$ due to a (poor) estimate of the number of trees, after appropriate combinatorial considerations, and $2^{k}$ due to the sum over the labels $\alpha_{v}= \pm$ ).

Therefore for $\beta=0$ the conjugating function $H_{\varepsilon}$ exists and it is uniformly continuous and uniformly bounded with a uniformly summable Fourier transform, for $\varepsilon$ (even complex) in $|\varepsilon|<\varepsilon_{0}(0) \stackrel{\text { def }}{=}(3 N)^{-3} F^{-1} 2^{-4}(1-\lambda)$, where an extra factor $2^{-1}$ has been inserted in order to obtain uniform bounds.

Taking $\beta>0$ requires estimating $|\boldsymbol{\nu}|^{\beta}$ : we bound it by $\sum_{v}\left|\boldsymbol{\nu}_{v}\right|^{\beta}$. Then from $\left|S_{0}^{-p(v)} \boldsymbol{\nu}_{v}\right| \leq N$ (otherwise the value vanishes), we infer that $\left|\boldsymbol{\nu}_{v}\right| \leq \lambda^{-|p(v)|} B N$, where $B \geq 1$ is a suitable constant. The $\operatorname{sum} \sum_{v}\left|\boldsymbol{\nu}_{v}\right|^{\beta}$ is over $k$ terms which can be estimated separately so that we can write $\sum_{v}\left|\boldsymbol{\nu}_{v}\right|^{\beta} \leq k\left|\boldsymbol{\nu}_{\bar{v}}\right|^{\beta}$ where $\left|\boldsymbol{\nu}_{\bar{v}}\right|=\max _{v}\left|\boldsymbol{\nu}_{v}\right|$. This can be taken into account by multiplying (3.14) by an extra factor $(B N)^{\beta} \lambda^{-\beta|p(\bar{v})|} \leq B N \lambda^{-\beta \sum_{v}\left|p_{v}\right|}$. Therefore if $\beta<1$ the $\sum_{\boldsymbol{\nu}}|\boldsymbol{\nu}|^{\beta}\left|h_{\alpha, \nu}^{(k)}\right|$ is bounded as in the case $\beta=0$ but the result is modified into

$$
\varepsilon_{0}(\beta)=(3 N)^{-3} F^{-1}\left(1-\lambda^{1-\beta}\right) 2^{-5} .
$$

This shows that $H_{\varepsilon}$ is analytic in $\varepsilon$ in the disk with radius $\varepsilon_{0}(\beta)$. Furthermore, since in (3.15) we inserted (for simplicity) an extra factor $2^{-1}$ in excess of the result obtained by the procedure described, the Hölder modulus is also uniformly bounded by a suitable function $C(\beta)$ of $\beta$. Note that $\varepsilon_{0}(\beta) \rightarrow 0$ for $\beta \rightarrow 1$.

The map $H_{\varepsilon}$ is a homeomorphism of $\mathbb{T}^{2}$ at fixed $\boldsymbol{\omega}$.

Remark: The result can also be interpreted as a "shadowing theorem". Consider the "noisy" trajectory $k \rightarrow \mathcal{S}_{\varepsilon}^{k} \mathbf{x}$ as a perturbation of a noiseless one. Then a noisy trajectory starting at $\mathbf{x}=(\boldsymbol{\omega}, x)$ will remain forever close to the trajectory of the point $H_{\varepsilon}^{-1}(\boldsymbol{\omega}, x)$ evolving under the noiseless motion. This is usually called a shadowing property.

\section{Heuristic considerations}

The notion of hyperbolicity or chaoticity can be extended, [16], to random systems, for a general map $\mathcal{S}$ acting on $\Omega \times \mathcal{F}$, as follows: (a) at every point $\mathbf{x}=(\boldsymbol{\omega}, x) \in \Omega \times \mathcal{F}$ the plane $W(x)$ tangent to $\mathcal{F}$ contains two planes, $W_{\boldsymbol{\omega}}^{s}(x), W_{\boldsymbol{\omega}}^{u}(x)$ with $W(x)=W_{\boldsymbol{\omega}}^{s}(x) \oplus W_{\boldsymbol{\omega}}^{u}(x)$, with positive dimensions $d^{s}, d^{u}$ and which are covariant, i.e. $\mathcal{S} W_{\boldsymbol{\omega}}^{a}(x)=W_{\tau \boldsymbol{\omega}}^{a}\left(f_{\boldsymbol{\omega}}(x)\right)$;

(b) for some $C, \kappa>0$ :

$$
\begin{aligned}
&\left\|\partial_{x} f_{\boldsymbol{\omega}}^{* k}(x) v\right\| \leq C e^{-k \kappa}\|v\|, v \in W_{\boldsymbol{\omega}}^{s}(x), \\
&\left\|\partial_{x} f_{\boldsymbol{\omega}}^{*(-k)}(x) v\right\| \leq C e^{-k \kappa}\|v\|, \quad v \in W_{\boldsymbol{\omega}}^{u}(x),
\end{aligned}
$$

for all $k>0 ; f_{\boldsymbol{\omega}}^{* k}$ is defined below (1.4) and the sizes $\|\cdot\|$ of the vectors are evaluated in the metrics at the points to which they are applied (i.e. $f_{\boldsymbol{\omega}}^{* k}(x)$ or $x$ or $\left.f_{\boldsymbol{\omega}}^{*(-k)}(x)\right)$; (c) $W_{\boldsymbol{\omega}}^{a}(x)$ are continuous in $\boldsymbol{\omega}, x$.

In the example (1.3) with $\varepsilon=0$ one has $\partial_{x}\left(f_{\omega}^{*( \pm k)}\right)=$ $S_{0}^{ \pm k}$ so that properties (a,b,c) are satisfied, trivially, because the map considered is an Anosov map.

It is also natural to define the expansion and contraction rates at $(\boldsymbol{\omega}, x)$. They will simply be

$$
J_{\boldsymbol{\omega}, N}^{a}(x)=\log \left|\left(\operatorname{det} \frac{\partial f_{\boldsymbol{\omega}}^{*(a N)}(x)}{\partial x}\right)\right|_{a}, \quad a=u, s,
$$

where $(a N)$ means $N$ if $a=u$ and $-N$ if $a=s$ while the label $a$ appended to the Jacobian matrix means that it is regarded as acting as a map from the plane $W_{\boldsymbol{\omega}}^{a}(x)$ to the plane $W_{\tau^{N} \boldsymbol{\omega}}^{a}\left(f_{\boldsymbol{\omega}}^{* N}(x)\right)$.

Note that the above expansion rates are not intrinsic geometric objects as they depend on the metric used on the manifold, as also the constants $C, \kappa$ do.

The volume measure $\mu_{0}(\mathrm{~d} x)$ on $\mathcal{F}$ can be visualized, fixed $\boldsymbol{\omega} \in \Omega$, in terms of the above rates: imagine to fix, for every point $x \in \mathcal{F}$, two segments located with center in $x$ and lying on the unstable or on the stable planes through $x$, and call them $\Delta_{\boldsymbol{\omega}}^{u}(x)$ and $\Delta_{\boldsymbol{\omega}}^{s}(x)$, respectively. Then we can build a tiny rectangle through $x$ by considering the line elements through $x$

$$
\begin{aligned}
& \delta_{u}(N, x)=f_{\tau^{N} \boldsymbol{\omega}}^{*(-N)}\left(\Delta_{\tau^{N} \boldsymbol{\omega}}^{u}\left(f_{\boldsymbol{\omega}}^{* N}(x)\right)\right), \\
& \delta_{s}(N, x)=f_{\tau^{-N} \boldsymbol{\omega}}^{* N}\left(\Delta_{\tau^{-N} \boldsymbol{\omega}}^{s}\left(f_{\boldsymbol{\omega}}^{*(-N)}(x)\right)\right),
\end{aligned}
$$

and by drawing through each point $y \in \delta_{s}$ the line element $\delta^{u}(N, x)$ and through each point of $y \in \delta_{s}$ the line element $\delta^{s}(N, x)$ and collecting the intersections: this is well defined, and will be called $\delta_{\boldsymbol{\omega}, N}(x)$. This is close to a rectangle with volume exponentially small as $N \rightarrow \infty$ and, to leading order in $N$, given by

$$
\operatorname{vol} \delta_{\boldsymbol{\omega}, N}(x)=e^{-J_{\boldsymbol{\omega}, N}^{u}(x)} e^{J_{\boldsymbol{\omega}, N}^{s}(x)} \gamma_{\boldsymbol{\omega}}(x),
$$

with, if $\alpha_{\boldsymbol{\omega}}(x)$ equals the angle between the stable and unstable planes $W_{\boldsymbol{\omega}}^{a}(x)$ at $x$,

$$
\gamma_{\boldsymbol{\omega}}(x) \stackrel{\text { def }}{=} \cos \alpha_{\boldsymbol{\omega}}(x) \prod_{a=u, s}\left|\Delta_{\tau^{(a N)}}^{a} \boldsymbol{\omega}\left(f_{\boldsymbol{\omega}}^{*(a N)}(x)\right)\right|,
$$

where $(a N)$ has the meaning explained after (4.2). The function $\gamma_{\boldsymbol{\omega}}(x)$ is uniformly bounded away from 0 and 
$\infty$. Hence the ratio of the volumes of two such parallelepipeds, centered at $x$ and $y$, can be computed by the ratio between the contraction factors in (4.3) evaluated at the two points (because the basic surface elements $\Delta_{\omega}^{a}(x)$ have all the same size and shape up to a factor $\left.\gamma_{\boldsymbol{\omega}}(x) / \gamma_{\boldsymbol{\omega}}(y)\right)$.

A consequence is that if we attribute to each such parallelepiped a measure proportional to

$$
e^{-J_{\boldsymbol{\omega}, 2 N}^{u}\left(f_{\boldsymbol{\omega}}^{*(-N)} x\right)},
$$

then we expect that in the limit as $N \rightarrow \infty$ the probability distribution has a limit $\mu_{\boldsymbol{\omega}}(\mathrm{d} x)$ such that $\mu_{\boldsymbol{\omega}}(\mathrm{d} x) P(\mathrm{~d} \boldsymbol{\omega}) \stackrel{\text { def }}{=} \mu(\mathrm{d} x \mathrm{~d} \boldsymbol{\omega})$ is an invariant distribution for the system $\left(\Omega \times \mathcal{F}, \mathcal{S}_{\varepsilon}\right)$ which should describe the statistical properties of almost all data initially chosen with the distribution $\mu_{0}(\mathrm{~d} x) P(\mathrm{~d} \boldsymbol{\omega})$ : i.e. $\mu(\mathrm{d} x \mathrm{~d} \boldsymbol{\omega})$ should be the $S R B$ distribution. Likewise replacing in (4.6) $N$ with $-N$ and $J^{u}$ with $-J^{s}$ one should obtain the SRB distribution for the backwards motion (i.e. for the map $\mathcal{S}^{-1}$ ).

\section{Overshadowing}

Therefore we look for an explicit algorithm to construct a useful representation of (4.6) or, what is the same, for the function $J_{\boldsymbol{\omega}, 1}(x)$ because

$$
J_{\boldsymbol{\omega}, 2 N}^{u}\left(f_{\boldsymbol{\omega}}^{*(-N)} x\right) \equiv \sum_{j=-N}^{N} J_{\boldsymbol{\omega}, 1}^{u}\left(f_{\boldsymbol{\omega}}^{* j}(x)\right),
$$

by the chain differentiation rule for composition of functions and by the multiplication rules of determinants of matrices.

We shall use the notations of Sec.3, where the conjugation $H_{\varepsilon}$, transforming a perturbation of the map $\mathcal{S}_{\varepsilon}(\boldsymbol{\omega}, x)=\left(\tau \boldsymbol{\omega}, S_{0} x+\varepsilon \psi_{\boldsymbol{\omega}}(x)\right)$ of the torus $\Omega \times \mathbb{T}^{2}$ into a noiseless map $\mathcal{S}_{0}(\boldsymbol{\omega}, x)=\left(\tau \boldsymbol{\omega}, S_{0} x\right)$, has been derived. The homeomorphism $\left(\boldsymbol{\omega}^{\prime}, x^{\prime}\right) \longleftrightarrow\left(\boldsymbol{\omega}, x+h_{\boldsymbol{\omega}}(x)\right) \equiv$ $H_{\varepsilon}(\boldsymbol{\omega}, x)$ can be used not only to construct the dynamics but also the stable and unstable manifolds of each point. The latter manifolds through $\left(\boldsymbol{\omega}, x+h_{\boldsymbol{\omega}}(x)\right)$ are given by parametric equations of the form

$$
\gamma_{\alpha}(t)=\left(\boldsymbol{\omega}, h_{\boldsymbol{\omega}}\left(x+t v_{\alpha}\right)\right) \quad t \in \mathbb{R}, \quad \alpha= \pm,
$$

where $t \rightarrow x+t v_{\alpha}$ is the unstable or stable manifold for the unperturbed map $S_{0}$ of $\mathbb{T}^{2}$ into itself, respectively if $\alpha=+$ or $\alpha=-$.

The construction of Sec.3 gives a Hölder continuous conjugation with a prefixed exponent $\beta<1$ for a perturbation strength $\varepsilon$ that is suitably small, depending on how close $\beta$ is to 1 . However, in general, the conjugation is not be differentiable: therefore (5.2) cannot be used to compute the derivatives appearing in the Jacobians $J_{\boldsymbol{\omega}, 1}^{a}(x)$ and the parametrization (5.2) is not very useful.

Instead of constructing the stable an unstable manifolds parameterized so that the required derivatives appearing in (5.1) can be computed (or just shown to exist) we remark that all we need are the expansion coefficients in the stable and unstable directions of any point $(\boldsymbol{\omega}, x)$.

To find them we apply once more the technique discussed in the previous two sections. Calling $\widehat{\Theta}$ the (noncompact) space $\Omega \times \mathbb{T}^{2} \times \mathbb{R}^{2}$ we define on it the dynamical system $\left(\widehat{\Theta}, \widehat{\mathcal{S}}_{0}\right)$ with

$$
\widehat{\mathcal{S}}_{0}(\boldsymbol{\omega}, x, v)=\left(\tau \boldsymbol{\omega}, S_{0} x, S_{0} v\right),
$$

where $v$ has to be interpreted as a tangent vector to $\mathbb{T}^{2}$ in $x$ and $S_{0}$ is the action of the derivative of the map $x \rightarrow S_{0} x$ on the tangent vector $v$. Since the map $S_{0}$ is locally linear there is no point in distinguishing between the constant matrix $\partial_{x} S_{0} x$ and the map $S_{0}$ so that we indulge in the abuse of notation implicit in (5.3).

We can consider the perturbation of $\widehat{\mathcal{S}}_{0}$ defined by

$$
\begin{aligned}
& \widehat{\mathcal{S}}_{\varepsilon}(\boldsymbol{\omega}, x, v)= \\
& =\left(\tau \boldsymbol{\omega}, S_{0} x+\varepsilon \psi_{\boldsymbol{\omega}}(x), S_{0} v+\varepsilon\left(v \cdot \partial_{x}\right) \psi_{\boldsymbol{\omega}}(x)\right),
\end{aligned}
$$

where the third term is the vector $v$ tranformed by the map $x^{\prime}=x+\varepsilon \psi_{\boldsymbol{\omega}}(x)$ into a tangent vector at $x^{\prime}$.

For uniformity of notation in what follows, we shall denote $S_{\boldsymbol{\omega}}(x)=S_{0} x+\varepsilon \psi_{\boldsymbol{\omega}}(x)$ instead of $f_{\boldsymbol{\omega}}(x)$ as done so far.

This system fails to be an Anosov system not only because of the noise (which acts trivially, however) but also because the space $\Omega \times \mathbb{T}^{2} \times \mathbb{R}^{2}$ is not compact. Nevertheless we can still try to find an isomorphism between $\widehat{\mathcal{S}}_{\varepsilon}$ and $\widehat{\mathcal{S}}_{0}$.

Success would mean that it is possible to decouple from the noise also the evolution of the tangent vectors, i.e. of the infinitesimal displacements: not only we could match individual trajectories in the perturbed and in the unperturbed system, as done in Sec.3, but we could even achieve "shadowing" of infinitesimally close pairs of trajectories which would split apart at the same rates as the unperturbed ones. If possible this property could be called overshadowing: but not surprisingly this turns out to be in general impossible (as it will be implicit in what follows). The next simplest goal, in trying to compare infinitesimally close pairs of trajectories, is to try to conjugate $\widehat{\mathcal{S}}_{\varepsilon}$ with a map $\widehat{\mathcal{S}}_{0, \varepsilon}$ defined by

$$
\widehat{\mathcal{S}}_{0, \varepsilon}(\boldsymbol{\omega}, x, v)=\left(\tau \boldsymbol{\omega}, S_{0} x, S_{0} v+\Gamma_{\boldsymbol{\omega}}(x) v\right),
$$

with $\Gamma_{\boldsymbol{\omega}}(x)$ a $2 \times 2$-matrix commuting with $S_{0}$ (hence diagonal on the basis $v_{ \pm}$), analytic in $\varepsilon$ and vanishing at $\varepsilon=0$. Therefore we look for a map $\widehat{H}_{\varepsilon}$ of a simple form and such that $\widehat{\mathcal{S}}_{\varepsilon} \circ \widehat{H}_{\varepsilon}=\widehat{H}_{\varepsilon} \circ \widehat{\mathcal{S}}_{0, \varepsilon}$, i.e.

$$
\left.\widehat{H}_{\varepsilon}:(\boldsymbol{\omega}, x, w) \longleftrightarrow\left(\boldsymbol{\omega}, x+h_{\boldsymbol{\omega}}(x)\right), w+K_{\boldsymbol{\omega}}(x) w\right),
$$

where $K$ is a $2 \times 2$-matrix analytic in $\varepsilon$ and 0 at $\varepsilon=0$, to be determined. In guessing (5.6), advantage is taken of the already known conjugation $H_{\varepsilon}$ between $\mathcal{S}_{\varepsilon}$ and $\mathcal{S}_{0}$, from the analysis of Sec.3, giving us the function $h_{\boldsymbol{\omega}}(x)$. 
Remarks: (1) Let $\mathcal{K}_{\boldsymbol{\omega}}(x)=1+K_{\boldsymbol{\omega}}(x)$ and let $\mathcal{L}_{\boldsymbol{\omega}}(x)=$ $S_{0}+\Gamma_{\boldsymbol{\omega}}(x)$ be functions with values in the $2 \times 2$ matrices. Then the conjugation (5.6) is equivalent to the following equation

$$
\partial_{x} S_{\boldsymbol{\omega}}\left(H_{\varepsilon}(\boldsymbol{\omega}, x)\right) \mathcal{K}_{\boldsymbol{\omega}}(x) v=\mathcal{K}_{\tau \boldsymbol{\omega}}\left(S_{0} x\right) \mathcal{L}_{\boldsymbol{\omega}}(x) v
$$

and to (3.3) for $H_{\varepsilon}$, which is an equation independent of (5.7) (already solved in Sect.3). This implies that the vector $w_{\boldsymbol{\omega}, \pm}(x)=\mathcal{K}_{\boldsymbol{\omega}}(x) v_{ \pm}$satisfies:

$$
\begin{aligned}
& \partial_{x} S_{\boldsymbol{\omega}}\left(H_{\boldsymbol{\omega}}(x)\right) w_{\boldsymbol{\omega}, \pm}(x)=\lambda_{\boldsymbol{\omega}, \pm}(x) w_{\tau \boldsymbol{\omega}, \pm}\left(S_{0} x\right)= \\
& =\left(\lambda_{ \pm}+\gamma_{ \pm, \boldsymbol{\omega}}(x)\right) w_{\tau \boldsymbol{\omega}, \pm}\left(S_{0} x\right)
\end{aligned}
$$

where $\lambda_{\boldsymbol{\omega}, \pm}(x)$ are the diagonal elements of $\mathcal{L}_{\boldsymbol{\omega}, \varepsilon}(x)$.

(2) The conjugation defined in (5.6) is the right one to look at the stable and unstable directions. Its solution gives the stable and unstable directions as functions of the unperturbed point $H_{\varepsilon}^{-1}(\boldsymbol{\omega}, x)$.

(3) Equation (5.7) does not determine $\mathcal{K}_{\boldsymbol{\omega}}(x)$ uniquely. Indeed, if $l_{\boldsymbol{\omega}, \pm}(x)$ are two non-zero functions from $\mathbb{T}^{2}$ to $\mathbb{R}$ and if $\lambda_{\boldsymbol{\omega}, \pm}(x), w_{\boldsymbol{\omega}, \pm}(x)$ solve (5.8), also

$$
\begin{aligned}
& \bar{\lambda}_{\boldsymbol{\omega}, \pm}(x)=\frac{l_{\boldsymbol{\omega}, \pm}(x)}{l_{\tau \boldsymbol{\omega}, \pm}\left(S_{0} x\right)} \lambda_{\boldsymbol{\omega}, \pm}(x), \\
& \bar{w}_{\boldsymbol{\omega}, \pm}(x)=l_{\boldsymbol{\omega}, \pm}(x) w_{\boldsymbol{\omega}, \pm}(x)
\end{aligned}
$$

solve it. To fix this ambiguity we will require that the diagonal elements of $K_{\boldsymbol{\omega}}(x)$, on the basis $v_{ \pm}$, are equal to 0 , i.e. the matrix $K_{\boldsymbol{\omega}}(x)$ is completely off-diagonal.

To simplify the rather heavy notations we denote

$$
\begin{aligned}
& \psi_{\alpha}(\boldsymbol{\omega}, x) \equiv \psi_{\boldsymbol{\omega}, \alpha}(x), \quad H(\boldsymbol{\omega}, x) \equiv\left(\boldsymbol{\omega}, x+h_{\boldsymbol{\omega}}(x)\right) \\
& \Gamma(\boldsymbol{\omega}, x) \equiv \Gamma_{\boldsymbol{\omega}}(x), \quad K(\boldsymbol{\omega}, x) \equiv K_{\boldsymbol{\omega}}(x), \\
& \xi \equiv \mathbf{x}=(\boldsymbol{\omega}, x),
\end{aligned}
$$

and the equation that the matrices $K(\xi), \Gamma(\xi)$ have to satisfy is a transcription of (5.7)

$$
\begin{aligned}
& \left(S_{0} K(\xi)-K\left(\mathcal{S}_{0} \xi\right) S_{0}\right)_{i j}= \\
& =-\varepsilon \partial_{x_{j}} \psi_{i}(H(\xi))-\varepsilon \partial_{x_{s}} \psi_{i}(H(\xi)) K(\xi)_{s j}+ \\
& +\Gamma(\xi)_{i j}+\left(K\left(\mathcal{S}_{0} \xi\right) \Gamma(\xi)\right)_{i j}
\end{aligned}
$$

where $\partial_{x}$ denotes a derivative of $\psi$ with respect to its original argument and repeated indices mean implicit summation (to abridge notations).

We write the above matrix equation on the basis in which $S_{0}$ and $\Gamma$ are diagonal, i.e. on the basis formed by the two eigenvectors $v_{ \pm}$of $S_{0}$ in which the matrices $K_{\boldsymbol{\omega}}, \Gamma_{\boldsymbol{\omega}}$ have been assumed to take the form

$$
\begin{aligned}
\Gamma(\xi) & =\left(\begin{array}{cc}
\gamma_{+}(\xi) & 0 \\
0 & \gamma_{-}(\xi)
\end{array}\right), \\
K(\xi) & =\left(\begin{array}{cc}
0 & k_{+}(\xi) \\
k_{-}(\xi) & 0
\end{array}\right) .
\end{aligned}
$$

If $\alpha= \pm, \beta=-\alpha$ and $\partial_{\alpha} \stackrel{\text { def }}{=} v_{\alpha} \cdot \partial_{\varphi},(5.11)$ becomes for $i=j$

$$
\begin{aligned}
0= & -\varepsilon \partial_{\alpha} \psi_{\alpha}(H(\xi))-\varepsilon K_{\beta, \alpha}(\xi) \partial_{\beta} \psi_{\alpha}(H(\xi))+ \\
& +\gamma_{\alpha}(\xi)
\end{aligned}
$$

and, for $i \neq j$ and if $\lambda_{+}=\lambda^{-1}, \lambda_{-}=-\lambda$ are the eigenvalues of $S_{0}($ with $\lambda=(\sqrt{5}-1) / 2)$,

$$
\begin{aligned}
& \left(\lambda_{\alpha} K_{\alpha, \beta}(\xi)-\lambda_{\beta} K_{\alpha, \beta}\left(\mathcal{S}_{0} \xi\right)=\right. \\
& \quad-\varepsilon \partial_{\beta} \psi_{\alpha}(H(\xi))-\varepsilon K_{\alpha, \beta}(\xi) \partial_{\alpha} \psi_{\alpha}(H(x))+ \\
& \quad+K_{\alpha, \beta}\left(\mathcal{S}_{0}(\xi)\right) \gamma_{\beta}(\xi) .
\end{aligned}
$$

If $\alpha^{\prime}=\frac{\alpha-1}{2}$, and $\alpha^{\prime \prime}=\frac{\alpha+1}{2}$ we can rewrite the equations (5.13) and (5.14) as

$$
\gamma_{\alpha}(\xi)=\varepsilon \partial_{\alpha} \psi_{\alpha}(H(\xi))+\varepsilon k_{\beta}(\xi) \partial_{\beta} \psi_{\alpha}(H(\xi)),
$$

and, respectively,

$$
\begin{aligned}
& k_{\alpha}(\xi)+\lambda^{2} k_{\alpha}\left(\mathcal{S}_{0}^{\alpha} \xi\right)=\alpha \lambda\left(-\varepsilon \partial_{\beta} \psi_{\alpha}\left(H\left(\mathcal{S}_{0}^{\alpha^{\prime}} \xi\right)\right)-(5.16)\right. \\
& \left.-\varepsilon k_{\alpha}\left(\mathcal{S}_{0}^{\alpha^{\prime}} \xi\right) \partial_{\alpha} \psi_{\alpha}\left(H\left(\mathcal{S}_{0}^{\alpha^{\prime}} \xi\right)\right)+k_{\alpha}\left(\mathcal{S}_{0}^{\alpha^{\prime \prime}} \xi\right) \gamma_{\beta}\left(\mathcal{S}_{0}^{\alpha^{\prime}} \xi\right)\right)
\end{aligned}
$$

These equations are in a form suitable for a recursive solution in powers of $\varepsilon$. For instance the first order is

$$
\begin{aligned}
& \gamma_{\alpha}^{(1)}(\xi)=\partial_{\alpha} \psi_{\alpha}(\xi), \quad \alpha= \pm \\
& k_{+}^{(1)}(\xi)+\lambda^{2} k_{+}^{(1)}\left(\mathcal{S}_{0} \xi\right)=-\lambda \partial_{-} \psi_{+}(\xi) \\
& k_{-}^{(1)}(\xi)+\lambda^{2} k_{-}^{(1)}\left(\mathcal{S}_{0}{ }^{-1} \xi\right)=\lambda \partial_{+} \psi_{-}\left(\mathcal{S}_{0}{ }^{-1} \xi\right),
\end{aligned}
$$

which has the solution

$$
\begin{aligned}
& \gamma_{\alpha}^{(1)}(\xi)=\partial_{\alpha} \psi_{\alpha}(\xi) \quad \alpha= \pm \\
& k_{+}^{(1)}(\xi)=-\lambda \sum_{q=0}^{\infty}(-1)^{q} \lambda^{2 q} \partial_{-} \psi_{+}\left(\mathcal{S}_{0}^{q} \xi\right) \\
& k_{-}^{(1)}(\xi)=\lambda \sum_{q=0}^{\infty}(-1)^{q} \lambda^{2 q} \partial_{+} \psi_{-}\left(\mathcal{S}_{0}^{-(q+1)} \xi\right)
\end{aligned}
$$

The equations for $\gamma_{\alpha}^{(k)}(\xi)$ and $k_{\alpha}^{(k)}(\xi)$ can be represented in graph form by suitably modifying the similar representation derived for $h_{\boldsymbol{\omega}, \alpha}^{(k)}(x)$ in Sec.3; compare Fig.2 with Fig.1.

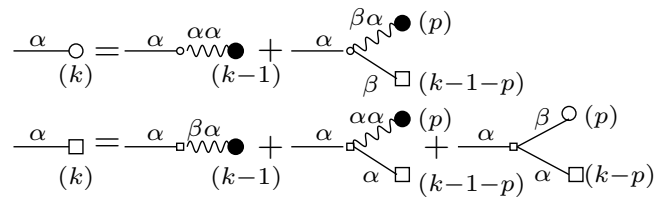

Fig. 2 Here $\alpha= \pm$ and $\beta=-\alpha$. All the lines have to imagined to carry arrows (not drawn) pointing toward the root. The line carrying a label $\alpha$ and emerging from a circle or a square with label $(k)$ denotes $\gamma_{\alpha}^{(k)}$ or $k_{\alpha}^{(k)}$, respectively. The wavy line emerging from a bullet with label $(p)$, with $1 \leq p<k-1$, ending in a small circle and carrying a pair of labels $\gamma, \delta$, represents $\left[\partial_{\gamma} \psi_{\delta}\right]^{(p)}(H(\xi))$, the $p$-th order in the power expansion in $\varepsilon$ of $\left(\partial_{\gamma} \psi_{\delta}\right)(H(\xi))$. The small square in the node closest to the root into which a wavy line 
arrives will carry a label $q=0,1,2 \ldots$ (see the first two graphs in the second line) and it expresses that $H\left(\mathcal{S}_{0}^{\alpha^{\prime}+\alpha \tilde{q}_{\alpha}}(\xi)\right)$ (in the second line), with $\tilde{q}_{+}=q$ and $\tilde{q}_{-}=q+1$, is the argument in which the functions $\partial_{\gamma} \psi_{\delta}$ are computed. Furthermore a summation over $q=0,1, \ldots$ and a multiplication by $-\alpha(-1)^{q} \lambda^{1+2 q}$ is understood to be performed over the nodes represented as small squares. The last graph in the second line does not carry any further labels and it represents the last term in (5.16).

The representation is drawn in figure Fig.2 and the symbols are explained in the corresponding caption: the reader will recognize in them a pictorial rewriting of (5.16).

In this case too we can continue the expansion until in the r.h.s. of Fig.2 all top nodes of the graph are either squares or circles carrying a label (1), i.e. they represent a first order contribution to $\Gamma$ (circle) or to $K$ (square), or bullets representing either $\partial_{\alpha} \psi_{\beta}(\xi)$ or $\partial_{\alpha} \psi_{\beta}\left(\mathcal{S}_{0}^{\alpha^{\prime}+\alpha \tilde{q}_{\alpha}} \xi\right)$; cf. the caption of Fig.2 for notations. Of course the latter quantities can themselves be represented by the tree expansion discussed in Sec.3: if we do so then we obtain a full expansion in powers of $\varepsilon$ in which the wavy lines with label $p$ are replaced by a tree with $p$ nodes.

The rule to construct the value of each tree graph is easily read from (5.11) and from the rules discussed above, and in Sec.3, to build the value of trees represent$\operatorname{ing} h$.

The estimate of the $k$-th order contribution is given by (3.14) with an extra factor $N^{k}$ to take into account the extra derivatives due to the lines with two labels. Also the counting of the trees has to be modified but the end result will be that $\Gamma$ and $K$ are expressed by convergent series in $\varepsilon$ for $|\varepsilon|<\varepsilon_{0}(\beta)$, where $\varepsilon_{0}(\beta)$ can be taken of the form (3.15) with a different numerical factor and with $N$ replaced by $N^{2}$.

\section{Construction of the SRB distribution}

Consider the partition $\mathcal{E}$ of $\Omega \times \mathcal{F}$ by the sets $E_{\omega, \sigma}=C_{\omega} \times$ $P_{\sigma}$ where $\mathcal{C}=\left\{C_{1}, \ldots, C_{m}\right\}$ is the partition of $\Omega$ according to the value of the symbol $\omega_{0}$ and $\mathcal{P}_{0}=\left\{P_{1}, \ldots, P_{n}\right\}$ is a Markovian pavement of $\mathcal{F}$ for the map $S_{0},[8]$. It is convenient to suppose that the Markovian pavement $\mathcal{P}_{0}$ is "reversible". This means that there exists a map $I_{0}$, with the property that $I_{0}^{2}= \pm 1$ and $I_{0} \circ S_{0}^{k}=S_{0}^{-k} \circ I_{0}$ for some integer $k$, such that for all $\sigma=1, \ldots, n$ one has $I_{0} P_{\sigma}=P_{\sigma^{T}}$ for some $\sigma^{T}$ (this is not restrictive as it can be achieved by considering the new pavement $\left.I_{0} \mathcal{P}_{0} \cap \mathcal{P}_{0}\right)$. Given a point $\xi$ let $(\boldsymbol{\omega}, \boldsymbol{\sigma})$ be its "history" on the partition $\mathcal{E}$ for the map $\mathcal{S}_{0}$. This means that $\mathcal{S}_{0}^{k} \xi \in E_{\omega_{k}, \sigma_{k}}$ for all $k$. In terms of the sequences $(\boldsymbol{\omega}, \boldsymbol{\sigma})$ the motion is simply a translation $(\tau, \rho)$ such that $(\tau \boldsymbol{\omega}, \rho \boldsymbol{\sigma})_{j}=(\boldsymbol{\omega}, \boldsymbol{\sigma})_{j+1}$, [8].

The partition $\mathcal{E}^{\prime}=H_{\varepsilon}(\mathcal{E})$ is then Markovian for the perturbed system. A correspondence can be generated between points in $\Omega \times \mathcal{F}$ by associating two points $\xi^{\prime}$ and $\xi$ if the first has history $(\boldsymbol{\omega}, \boldsymbol{\sigma})$ on $\mathcal{E}^{\prime}$ identical to the history of $\xi$ on the partition $\mathcal{E}$ under the action of the map
$\mathcal{S}_{0}$ : we denote $\xi^{\prime}=X_{\varepsilon}(\boldsymbol{\omega}, \boldsymbol{\sigma})$ and $\xi=X_{0}(\boldsymbol{\omega}, \boldsymbol{\sigma})$. The correspondence so defined is identical to the correspondence between $\xi^{\prime}$ and $\xi$ defined by $\xi^{\prime}=H(\xi)$. And in Sec. 5 it has been proved that

There exists $\varepsilon_{0}(\beta)$ such that the expansion rate $\lambda_{u, \boldsymbol{\omega}}(\boldsymbol{\sigma})$ of $\mathcal{S}_{\varepsilon}$ along the unstable manifold of $\xi^{\prime}$ is defined and holomorphic in $\varepsilon$ in the disk $|\varepsilon|<\varepsilon_{0}(\beta)$. As a function of $(\boldsymbol{\omega}, \boldsymbol{\sigma})$ it is Hölder continuous with some exponent $0<$ $\beta<1$ and modulus $C(\beta)$.

This can be seen as follows: one just notes that if $\xi^{\prime}=H_{\varepsilon}(\xi)$ then the unstable direction at $\xi^{\prime}$, which is a vector tangent to $\mathcal{F}$, will be $w_{\boldsymbol{\omega},+}(x)=v_{+}+K_{\boldsymbol{\omega}}(x) v_{+}$. Then the expansion rate along the unstable manifold of $\xi^{\prime}=\left(\boldsymbol{\omega}, x^{\prime}\right)=H_{\varepsilon}(\xi)$ will be $e^{A_{\boldsymbol{\omega}, u}(\boldsymbol{\sigma}) \stackrel{\text { def }}{=}}\left|J_{\boldsymbol{\omega}, 1}^{u}\left(x^{\prime}\right)\right|$ and, by $(5.8),(4.1),(4.2)$,

$$
A_{\boldsymbol{\omega}, u}(\boldsymbol{\sigma})=\log \left(\left(\lambda_{+}+\gamma_{\boldsymbol{\omega},+}(x)\right) \frac{\left|w_{\boldsymbol{\omega},+}\left(S_{0} x\right)\right|}{\left|w_{\boldsymbol{\omega},+}(x)\right|}\right),
$$

where $\left|w_{\boldsymbol{\omega},+}(x)\right|=\sqrt{1+k_{\boldsymbol{\omega},+}(x)^{2}}$.

The functions $\gamma_{\boldsymbol{\omega},+}, k_{\boldsymbol{\omega},+}$ are analytic in $\varepsilon$ and Hölder continuous in $\xi$ with a uniformly bounded modulus $C(\beta)$ if $\beta<1$ and $\varepsilon$ is small enough. Since $\left\{\left(\omega_{i}, \sigma_{i}\right)\right\}_{i=-\infty}^{\infty}$ fixed means $\xi$ fixed (because $\boldsymbol{\sigma}$ is the history of a point $x$ on a Markov pavement for the $\varepsilon$-independent map $S_{0}$ ), we see that $A_{\boldsymbol{\omega}, u}(\boldsymbol{\sigma})$ is analytic in $\varepsilon$ at fixed $(\boldsymbol{\omega}, \boldsymbol{\sigma})$ and Hölder continuous in $(\boldsymbol{\omega}, \boldsymbol{\sigma})$ and therefore in $\xi$.

Hence the function $A_{\boldsymbol{\omega}, u}(\boldsymbol{\sigma})$ generates a short range potential on the one-dimensional "spin system" whose states are the sequences $(\boldsymbol{\omega}, \boldsymbol{\sigma})$, in the sense of [14].

An identical argument holds for the similar function $A_{\boldsymbol{\omega}, s}(\boldsymbol{\sigma}) \stackrel{\text { def }}{=} \log \left|J_{\boldsymbol{\omega}, 1}^{s}\left(x^{\prime}\right)\right|$. It follows, by standard arguments on one-dimensional Gibbs distributions, see Chap.6 in [8], that the "short range" Gibbs distribution $\nu$ with formal energy function

$$
\sum_{k=-\infty}^{0} A_{\tau^{k} \boldsymbol{\omega}, s}\left(\rho^{k} \boldsymbol{\sigma}\right)+\sum_{k=0}^{\infty} A_{\tau^{k} \boldsymbol{\omega}, u}\left(\rho^{k} \boldsymbol{\sigma}\right)
$$

is well defined and it has the property that if $C_{\boldsymbol{\omega}, \boldsymbol{\sigma}}^{N}$ is defined as $\left\{\boldsymbol{\omega}^{\prime}, \boldsymbol{\sigma}^{\prime}\left|\omega_{i}^{\prime}=\omega_{i}, \sigma_{i}^{\prime}=\sigma_{i}, \forall\right| i \mid \leq N\right\}$, then

$$
B^{-1} \leq \frac{\nu\left(C_{\boldsymbol{\omega}, \boldsymbol{\sigma}}^{N}\right)}{\operatorname{vol}\left(C_{\boldsymbol{\omega}, \boldsymbol{\sigma}}^{N}\right)} \leq B
$$

for a suitable constant $B>0$.

Therefore the distribution $\nu$ is absolutely continuous with respect to the volume distribution (since $\gamma_{\omega}$ in (4.6) is bounded above and below).

The distribution $\nu$ is not translation-invariant because its Gibbs potential in the "far future" is governed by $A_{\boldsymbol{\omega}, u}(\boldsymbol{\sigma})$ while in the far past it is governed by $A_{\boldsymbol{\omega}, s}(\boldsymbol{\sigma})$, see (6.2). But short range Gibbs distributions (whether invariant or not) enjoy exponentially mixing properties: hence the distribution $\nu$, and consequently the volume 
distribution, will be very close on the evolution of the cylinders, for very large times, to the invariant Gibbs distributions with (formal) potentials

$$
\sum_{k=-\infty}^{+\infty} A_{\tau^{k} \boldsymbol{\omega}, a}\left(\rho^{k} \boldsymbol{\sigma}\right), \quad\left\{\begin{array}{l}
\text { as } p \rightarrow+\infty \text { if } a=u \\
\text { as } p \rightarrow-\infty \text { if } a=s
\end{array}\right.
$$

respectively. Therefore the distribution with potential in (6.4) with $a=u$ is the SRB distribution for the forward evolution while for $a=s$ the distribution is the SRB distribution for the backward evolution.

\section{Time reversal symmetry}

The result of Sec.6 allows us to conclude that the SRB distribution $\mu_{\text {srb }}$ will be a "space-time" Gibbs state for the energy function $A_{\boldsymbol{\omega}, u}(\boldsymbol{\sigma})$ in the sense of [14].

Time reversal symmetry is a symmetry that should be inherited from the microscopic time reversal symmetry of purely Hamiltonian dynamics. It may be destroyed by the thermostats, or better, by the models that are introduced to describe them.

In presence of noise the matter is even more involved. However under the assumptions that we have posed upon $\mathcal{S}_{\varepsilon}$ it is possible to show that if the noiseless system $\mathcal{S}_{0}$ is reversible a "kind" of time reversal symmetry will continue to hold when the noise is not neglected provided the noise also has a reversibility property.

We supposed, since the very beginning in Sec.1, reversibility of the stationary probability distribution $P$ on $\Omega$ in the sense that the operation $\theta$ defined by $\theta \boldsymbol{\omega}=\boldsymbol{\omega}^{T}$ with $\omega_{i}^{T}=\omega_{-i}$ ("history reversal") conserves the distribution $P$ :

$$
P\left(E^{T}\right)=P(E)
$$

where $E^{T}=\left\{\boldsymbol{\omega}^{T}: \boldsymbol{\omega} \in E\right\}$, for all (Borel-)sets $E$.

Suppose that the unperturbed system evolution on $\mathcal{F}$, that we assume to be an Anosov map $f$, is reversible in the sense that there is a map $I_{0}: \mathcal{F} \rightarrow \mathcal{F}$ with the property $I_{0}$ o $f^{k}=f^{-k}$ o $I_{0}$ for some $k$ and $I_{0}^{2}= \pm 1$, or more generally $I_{0}^{k^{\prime}}=1$ for some $k^{\prime}$ even.

The above assumption is satisfied in the examples treated in the previous sections because the time reversal operation $I_{0}\left(\varphi_{1}, \varphi_{2}\right)=\left(\varphi_{2},-\varphi_{1}\right)$ has the property $I_{0}$ o $S_{0}^{2}=S_{0}^{-2}$ о $I_{0}$, if $S_{0}$ is given by (1.1).

The problem is that it is not clear that a time reversal symmetry holds when there is a perturbation, even if small. But, if $\varepsilon$ is small, the time reversal symmetry $\mathcal{I}_{0}(\boldsymbol{\omega}, x)=\left(\boldsymbol{\omega}^{T}, I_{0} x\right)$ is not "broken" by the perturbation and the noisy system admits a time reversal symmetry $\mathcal{I}_{\varepsilon}$. The latter can be immediately written as

$$
\mathcal{I}_{\varepsilon} \stackrel{\text { def }}{=} H_{\varepsilon} \circ \mathcal{I}_{0} \circ H_{\varepsilon}^{-1} \text {. }
$$

However for the same reasons for which the conjugacy between $\mathcal{S}_{\varepsilon}$ and $\mathcal{S}_{0}$ was not smoother than Hölder continuous it will turn out that $\mathcal{I}_{\varepsilon}$ is also not necessarily smoother than Hölder continuous.
In terms of symbolic dynamic the time reversal $\mathcal{I}_{\varepsilon}$ acts on a point whose symbolic history is $(\boldsymbol{\omega}, \boldsymbol{\sigma})$ by transforming it into the point with symbolic history $\left(\boldsymbol{\omega}^{T}, \sigma^{T}\right)$, where $\left(\boldsymbol{\sigma}^{T}\right)_{i}=\sigma_{-i}^{T}$, if time reversal transforms the element $P_{\sigma}$ of the Markov pavement for $S_{0}$ into $P_{\sigma^{T}}$. It is therefore clear that $\mathcal{I}_{\varepsilon}^{k^{\prime}}=1$ if $I_{0}^{k^{\prime}}=1$.

The lack of smoothness of the new time reversal implies that the fluctuation theorem can certainly be proved only in cases in which the perturbed system also admits a smooth time reversal. Then the proof is the same as the one for Anosov maps because the latter was based on the existence of a Markovian partition of phase space and on the Gibbs property of the SRB distribution with a Gibbs weight generated by a function $A_{u, \boldsymbol{\omega}}(\boldsymbol{\sigma})$, as shown in Sec.6, satisfying $A_{u, \boldsymbol{\omega}^{T}}\left(\boldsymbol{\sigma}^{T}\right)=-A_{s, \boldsymbol{\omega}}(\boldsymbol{\sigma})$ (which follows from time reversal symmetry and from the isometric nature of such reversal) $[9,11]$.

Examples can be obtained easily: consider the paradigmatic example $\mathcal{S}_{\varepsilon}$ of the previous sections and the map $\mathcal{T}(\boldsymbol{\omega}, x, \boldsymbol{\eta}, y)$ defined on $\Omega \times \mathbb{T}^{2} \times \Omega \times \mathbb{T}^{2}$ by

$$
\mathcal{T}(\boldsymbol{\omega}, x, \boldsymbol{\eta}, y)=\left(\mathcal{S}_{\varepsilon}(\boldsymbol{\omega}, x), \mathcal{S}_{\varepsilon}^{-1}(\boldsymbol{\eta}, y)\right) .
$$

Then a time reversal symmetry (i.e. an isometry anticommuting with time and squaring to the identity) can be defined as $\mathcal{I}((\boldsymbol{\omega}, \boldsymbol{\eta}, x, y)=(\boldsymbol{\eta}, \boldsymbol{\omega}, y, x)$ and the fluctuation theorem applies to this system.

The last example is somewhat artificial: in applications time reversal should be a built-in symmetry so that its checking should be immediate. This is often the case in non-random systems. Note that in numerical simulations realizing the system $\mathcal{T}$ out of a system $\mathcal{S}$ would be easy.

\section{Structural stability}

All what has been said about the existence of the conjugacies to the unperturbed system can be extended to the cases in which the torus has arbitrary dimension and $S_{0}$ is an hyperbolic linear torsion (i.e. it is a map generated by a matrix $S_{0}$ with determinant \pm 1 , integer entries and all eigenvalues with modulus different from 1 ), by replacing $\lambda_{ \pm}$by the determinants of $S_{0}$ restricted to the stable or unstable planes.

Consider the case in which the map $x \rightarrow f(x)$ is just an Anosov map of a general manifold $\mathcal{F}$, still assuming analytic regularity. If the unperturbed Anosov map $f$ is close to some hyperbolic linear torsion $S_{0}$ of a torus it will be possible to find an analytic conjugacy to the systems to $\mathcal{S}_{0}$ simply because we can think that the perturbation $\varepsilon \psi_{\boldsymbol{\omega}}$ contains a non-random part which is the perturbation of $S_{0}$ into $f$. The same can be said of maps that can be analytically conjugated to hyperbolic torsions.

To extend the results on the fluctuation relation, however, the strong assumption about time reversal has to be always added.

More interesting and subtle is the further question of how much the above analysis depends on the assumption that we are perturbing a system close to one $\mathcal{S}_{0}$ in which 
a reversible noise and a linear torsion of a torus $S_{0}$ do not interact.

Under the only assumption that $S_{0}$ is analytic and Anosov the equations for $H$ will have to be written in coordinates and possibly several coordinate charts will have to be used. An apparently unsurmountable difficulty may seem that the directions $v_{ \pm}$will now depend on $x$ and therefore the solution algorithm will simply fail. A more attentive study of the equations shows, never- theless, that the equations are soluble order by order as at every order the curvature of the manifolds systematically gives contributions of higher order, see [1] for details showing that the existence of a convergent power series in $\varepsilon$ for $H$ exists. Since that is the main difficulty there is little doubt that the analysis that we have performed in detail for the case of the random perturbations of (1.1) carries unchanged to the last case.
[1] F. Bonetto, P. Falco, and A. Giuliani, Journal of Mathematical Physics 45, 3282 (2004).

[2] D. Ruelle, Journal of Statistical Physics 86, 935 (1997).

[3] J. Bricmont and A. Kupiainen, Nonlinearity 8, 379 (1995).

[4] M. Jiang and E. Mazel, Journal of Statistical Physics 82, 797 (1996).

[5] M. Jiang and Y. Pesin, Communications in Mathematical Physics 193, 675 (1998).

[6] J. Bricmont and A. Kupiainen, Communications in Mathematical Physics 178, 703 (1996).

[7] J. Bricmont and A. Kupiainen, Physica D 103, 18 (1997).

[8] G. Gallavotti, F. Bonetto, and G. Gentile, Aspects of the ergodic, qualitative and statistical theory of motion (Springer Verlag, Berlin, 2004).

[9] G. Gallavotti and E. Cohen, Physical Review Letters 74,
2694 (1995).

[10] G. Gallavotti and E. Cohen, Journal of Statistical Physics 80, 931 (1995).

[11] G. Gallavotti, Mathematical Physics Electronic Journal (MPEJ) 1, 1 (1995).

[12] J. Kurchan, Journal of Physics A 31, 3719 (1998).

[13] J. Lebowitz and H. Spohn, Journal of Statistical Physics 95, 333 (1999).

[14] C. Maes, Journal of Statistical Physics 95, 367 (1999).

[15] F. Bonetto, A. Kupiainen, and J. Lebowitz, Ergodic theory and dynamical systems pp. 59-88 (2005).

[16] L. Pei-Dong, Ergodic theory and dynamical systems 21, 1279 (2001).

$\mathrm{REV}_{\mathrm{T}} \mathrm{EX} \& \mathrm{E}_{\mathrm{Q}} \mathrm{A}_{\mathrm{L}} \mathrm{I}_{\mathrm{GN}} \mathrm{NO} \& \mathrm{~F}_{\mathrm{A}} \mathrm{N}_{\mathrm{H}} \mathrm{HR}$ 